\section{Dopamine Reuptake Transporter- Single-Photon Emission Computed Tomography and Transcranial Sonography as Imaging Markers of Prediagnostic Parkinson's Disease}

\author{
Alastair J. Noyce, MRCP, PhD, ${ }^{1,2}$ John Dickson, PhD, ${ }^{3}$ \\ Richard N. Rees, MRCP, ${ }^{4}$ Jonathan P. Bestwick, MSc, ${ }^{1}$ \\ loannis U. Isaias, MD, PhD, ${ }^{5}$ Marios Politis, MD, $\mathrm{PhD},{ }^{6}$ \\ Gavin Giovannoni, FRCP, PhD, ${ }^{7}$ \\ Thomas T. Warner, FRCP, PhD, ${ }^{2}$ \\ Andrew J. Lees, FRCP, MD, ${ }^{2}$ and \\ Anette Schrag, FRCP, $\mathrm{PhD}^{4 *}$
}

\begin{abstract}
${ }^{1}$ Preventive Neurology Unit, Wolfson Institute of Preventive Medicine, Barts and the London School of Medicine and Dentistry, Queen Mary University of London, London, UK ${ }^{2}$ Reta Lila Weston Institute and Department of Molecular Neuroscience, UCL Institute of Neurology, University College London, London, UK ${ }^{3} U C L$ Division of Medicine, University College London, London, UK ${ }^{4}$ Department of Clinical Neurosciences, Royal Free Campus, UCL Institute of Neurology, University College London, London, UK ${ }^{5}$ Department of Neurology, University Hospital Würzburg and Julius-MaximiliansUniversity, Würzburg, Germany ${ }^{6}$ Neurodegeneration Imaging Group, Maurice Wohl Clinical Neuroscience Institute, Kings College London, London, UK ${ }^{7}$ Blizard Institute, Barts and the London SMD, Queen Mary University of London, London, UK
\end{abstract}

\begin{abstract}
Objective: The objective of this study was to examine whether prediagnostic features of Parkinson's disease (PD) were associated with changes in dopamine reuptake transporter-single-photon emission computed tomography and transcranial sonography.

Methods: Prediagnostic features of PD (risk estimates, University of Pennsylvania Smell Identification Test,
\end{abstract}

This is an open access article under the terms of the Creative Commons Attribution License, which permits use, distribution and reproduction in any medium, provided the original work is properly cited.

${ }^{*}$ Corresponding author: Prof. Anette Schrag, Department of Clinical Neuroscience, UCL Institute of Neurology, Royal Free Campus, London NW3 2PF; a.schrag@ucl.ac.uk

Funding agency: This study was funded by Parkinson's UK (ref. F-1201) and an imaging grant from GE Healthcare.

Relevant conflicts of interests/financial disclosures: Nothing to report.

Received: 6 November 2017; Revised: 24 November 2017; Accepted: 27 November 2017

Published online 00 Month 2018 in Wiley Online Library (wileyonlinelibrary.com). DOI: 10.1002/mds.27282
Rapid Eye Movement Sleep Behavior Disorder Screening Questionnaire, and finger-tapping scores) were assessed in a large cohort of older U.K. residents. A total of 46 participants were included in analyses of prediagnostic features and MDS-UPDRS scores with the striatal binding ratio on dopamine reuptake transporter-single-photon emission computed tomography and nigral hyperechogenicity on transcranial sonography.

Results: The striatal binding ratio was associated with PD risk estimates $(P=.040)$, University of Pennsylvania Smell Identification Test $(P=.002)$, Rapid Eye Movement Sleep Behavior Disorder Screening Questionnaire scores $(P=.024)$, tapping speed $(P=.024)$, and MDSUPDRS motor scores $(P=.009)$. Remotely collected assessments explained $26 \%$ of variation in the striatal binding ratio. The inclusion of MDS-UPDRS motor scores did not explain additional variance. The size of the nigral echogenic area on transcranial sonography was associated with risk estimates $(P<.001)$ and MDSUPDRS scores $(P=.03)$ only.

Conclusions: The dopamine reuptake transportersingle-photon emission computed tomography results correlated with motor and nonmotor features of prediagnostic PD, supporting its potential use as a marker in the prodromal phase of PD. Transcranial sonography results also correlated with risk scores and motor signs. (C) 2018 The Authors. Movement Disorders published by Wiley Periodicals, Inc. on behalf of International Parkinson and Movement Disorder Society.

Key Words: Parkinson's disease; cohort; epidemiology; risk factors

Dopamine reuptake transporter (DAT)-single-photon emission computed tomography (SPECT) allows visualization of striatal dopaminergic innervation quantified through striatal binding ratios (SBR), and transcranial sonography (TCS) can be used to identify hyperechogenicity of the substantia nigra (SN). Reduced SBR with DAT-SPECT is present in most patients with Parkinson's disease (PD). Reduced SBR may also be seen in individuals with idiopathic anosmia and rapid eye movement sleep behavior disorder (RBD) and can predate the diagnosis of PD in these specific high-risk groups by 3 to 4 years. ${ }^{1-3} \mathrm{SN}$ hyperechogenicity is also present in most patients with PD, has been shown to be predictive of subsequent diagnosis of $\mathrm{PD}$, and is associated with some of the prediagnostic features of PD. ${ }^{4,5}$

It is unclear how early these imaging abnormalities occur in those who do not have a specific risk factor (such as anosmia or RBD) and whether changes are 
NOYCE ET AL

also present in individuals with other prediagnostic features of PD. We report cross-sectional associations of DAT-SPECT and TCS with several prediagnostic motor and nonmotor features in a prospective study to identify risk of PD in the general population.

\section{Methods}

The PREDICT-PD study assesses risk factors and prediagnostic features of PD collected prospectively online and uses an evidence-based algorithm to generate risk estimates annually. ${ }^{6}$ Participants aged 60 to 80 years at baseline completed an online assessment, including a computer keyboard tapping task (which records number of alternate finger taps in 30 seconds, termed kinesia score [KS]), and they were sent the University of Pennsylvania Smell Identification Test (UPSIT). Participants were ranked by risk scores, calculated from the results of a meta-analysis of risk factors and early features of PD (see Supplementary Material). ${ }^{7}$ The Queen Square Research Ethics Committee approved the study.

From the wider cohort, we invited $50 \%$ of participants with the top 5\% highest risk scores and 25 randomly selected participants across the middle and lower risk groups. Invitations were restricted to those younger than age 80 years at the time of scanning. Other exclusion criteria were those for DAT-SPECT and the main PREDICTPD study (see Supplementary Material). ${ }^{6}$

Participants were examined using the MDS-UPDRS part $3 .^{8}$ DAT-SPECT was performed using a GE Discovery 670 SPECT/CT machine (GE Healthcare, Chicago, Illinois). A standardized SPECT protocol was followed with images obtained approximately 3 hours after injection with $185 \mathrm{MBq}$ of ${ }^{123} \mathrm{I}$-2-b-carbomethoxy-3b-(4-iodophenyl)-N-(3-fluoropropyl) nortropane $\left({ }^{123} \mathrm{I}-\mathrm{FP}-\mathrm{CIT}\right)$ (GE Healthcare). ${ }^{9}$ For the semiquantitative analysis of tracer binding, BRASS software was used (HERMES Medical Solutions, Stockholm, Sweden). Volumes of interest (VOIs) were automatically defined to assess specific tracer binding in the striatum and over a reference region, the occipital cortex (OCC), to assess nonspecific binding. The count concentrations in these regions were used to calculate SBR as $\left.\left[\mathrm{VOI}_{\text {striatum }}-\mathrm{OCC}\right] / \mathrm{OCC}\right]$, where $\mathrm{VOI}_{\text {striatum }}$ and OCC were the count concentrations in the striatum and occipital cortex, respectively. An experienced image processor performed all semiquantitative analysis (JD) and 2 experts in the field of PD imaging (IUI and MP) reviewed and visually rated the DAT-SPECT images for each participant.

The TCS examination followed the standardized procedures described in the Supplementary Material. Given the operator and machine dependency of TCS, a validation study in patients with PD and controls was conducted to define relevant cut-offs before inclusion of PREDICT-PD participants (see Supplementary Material). The cut-off for $\mathrm{SN}$ hyperechogenicity that resulted from the validation stage was $0.16 \mathrm{~cm}^{2}$. The side with the greatest area of SN hyperechogenicity (SN-max) was used for the analysis. The presence of SN hyperechogenicity on the static images was also scored by an assessor (RNR) blinded to participant risk scores.

For the analysis, the outcome measures were the SBR for the side of the striatum with lower binding of tracer and SN-max for TCS. The exposure variables were PREDICTPD risk estimates (log odds of PD risk within 1 year of the scan date), UPSIT scores, Rapid Eye Movement Sleep Behavior Disorder Screening Questionnaire (RBDSQ) scores, and KS (alternate finger tapping) scores (previously referred to as intermediate markers ${ }^{6}$ ), and MDS-UPDRS motor scores. First, groupwise comparisons were undertaken between the highest risk and lower risk groups. Wilcoxon rank sum and $t$ tests were used depending on whether data were normally or nonnormally distributed. Fisher's exact test was used to analyze binary exposure/outcome associations. Linear regression was used to examine relationships between SBR and SN-max, and risk estimates, intermediate marker scores, and MDS-UPDRS scores. Univariate models and multivariate models were constructed to determine the combination of exposures that best predicted the outcome. Statistical analyses were undertaken using Stata (StataCorp, College Station, Texas).

\section{Results}

Of the 50 participants who underwent DAT-SPECT, 4 were excluded from the main analysis: 1 participant attended for imaging but had clinical features of PD, and 3 were scanned but had missing data for UPSIT, finger tapping, and/or recent risk data; 1 additional participant did not have TCS for technical reasons.

The median number of days between the closest survey date and the date of scan was 96 days (interquartile range, 34-136). Table 1 shows demographic and

TABLE 1. Differences between the higher and lower risk groups

\begin{tabular}{lccc}
\hline Factor & Higher risk & Lower risk & $P$ value \\
\hline $\mathrm{n}$ & 23 & 23 & \\
Median age in years (IQR) & $74.5(69.7-78.9)$ & $68.3(66.7-71.1)$ & $.003^{*}$ \\
Males (\%) & $23(100)$ & $16(70)$ & $.009^{\star \star}$ \\
Median risk (odds & $5.5(3.1-7.9)$ & $105(43-189)$ & $<.001^{*}$ \\
$\quad 1 / \mathrm{x}$ and IQR) & & & \\
Median UPSIT (IQR) & $28(25-33)$ & $34(29-36)$ & $.026^{\star}$ \\
Median RBDSQ (IQR) & $3(1-6)$ & $1(0-2)$ & $.007^{\star}$ \\
Mean KS (95\% CI) & $50.2(45.4-55.0)$ & $48.4(44.1-52.6)$ & $.559^{\star \star *}$ \\
Median MDS-UPDRS (IQR) & $3(2-7)$ & $1(0-2)$ & $.002^{*}$ \\
Mean worst SBR (95\% Cl) & $2.6(2.5-2.8)$ & $2.9(2.7-3.0)$ & $.071^{\star \star *}$ \\
Mean SN-max (95\% Cl) & $0.22(0.19-0.26)$ & $0.14(0.12-0.17)^{+}$ & $<.001^{* \star *}$
\end{tabular}

UPSIT, University of Pennsylvania Smell Identification Test; RBDSQ, Rapid Eye Movement Sleep Behavior Disorder Screening Questionnaire; KS, kinesia score (number of alternate keyboard taps in 30 seconds); MDS-UPDRS, Movement Disorders Society Unified Parkinson's Disease Rating Scale; SBR, striatal binding ratio; SN-max, area of substantia nigra hyperechogenicity on the most affected side; IQR, interquartile range; $95 \% \mathrm{CI}, 95 \%$ confidence interval.

*Wilcoxon rank sum test; ${ }^{\star \star}$ Fisher's exact test; ${ }^{\star \star \star}$ unpaired $t$ test. 

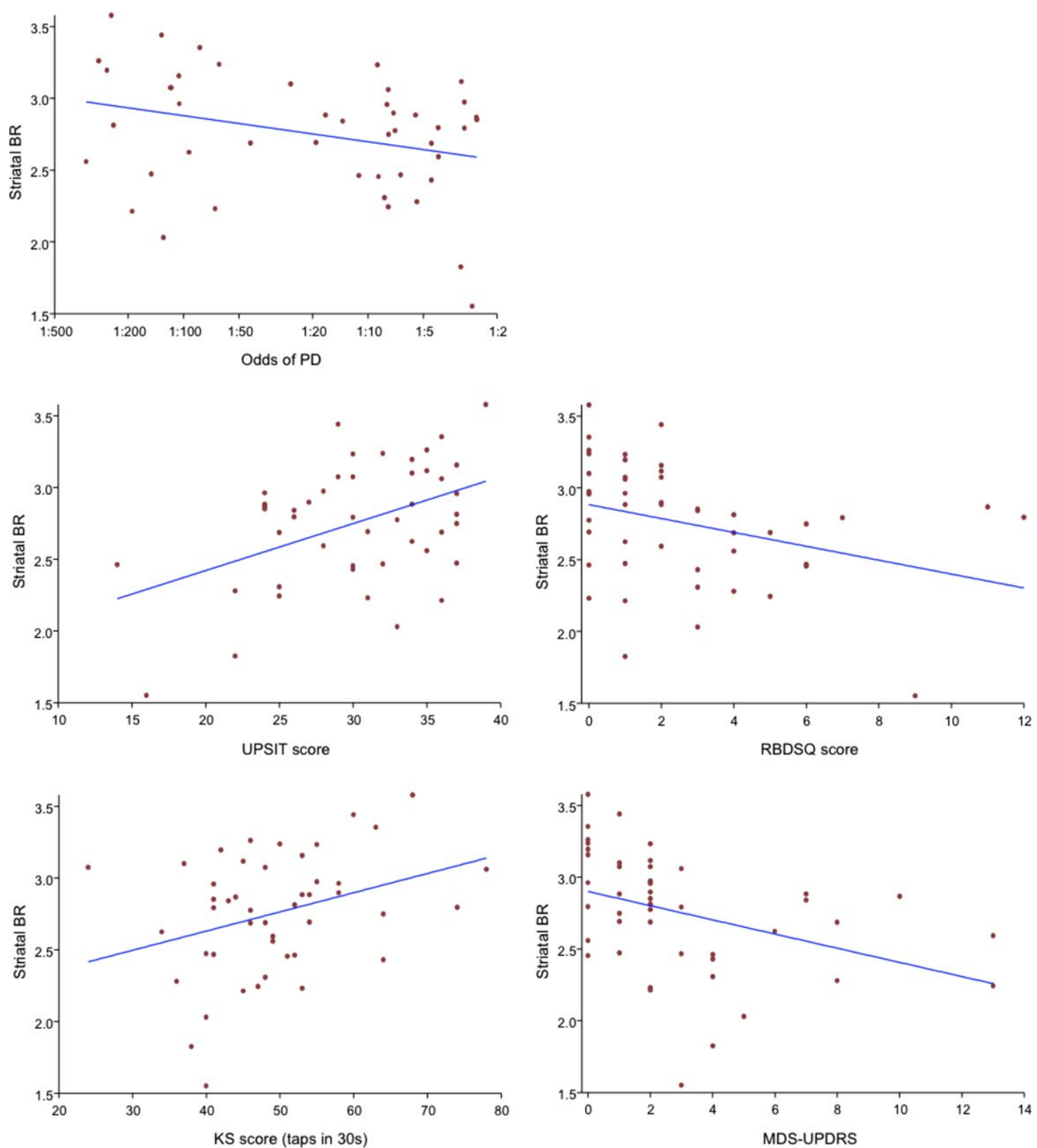

FIG. 1. Linear associations of risk scores and outcomes with striatal binding ratios. BR, binding ratio; UPSIT, University of Pennsylvania Smell Identification Test; RBDSQ, Rapid Eye Movement Sleep Behaviour Disorder Screening Questionnaire; KS, kinesia score (number of alternate keyboard taps in 30 seconds); MDS-UPDRS, Movement Disorders Society Unified Parkinson's Disease Rating Scale. [Color figure can be viewed at wileyonlinelibrary.com]

imaging data by risk group. Higher risk participants tended to have lower SBRs when compared with lower risk participants (SBR 2.64 vs $2.87, P=.071)$.

SBRs were associated with MDS-UPDRS motor scores $\left(r^{2}=0.13, P=.009\right)$, with the 3 intermediate outcomes (UPSIT $r^{2}=0.18, \quad P=.002 ; \quad$ RBDSQ $r^{2}=0.09, P=.024$; and $\left.\mathrm{KS} r^{2}=0.09, P=.024\right)$ and with PREDICT-PD risk estimates $\left(r^{2}=0.07, P=.040\right.$; see Fig. 1). The combination of PD risk score, RBDSQ, finger-tapping speed, and UPSIT explained a greater proportion of the variance in SBR than each of these variables alone $\left(r^{2}=0.26\right.$ vs $0.18 ; P=.050$ compared to UPSIT alone). Adding the MDS-UPDRS to the online assessments did not explain additional variance in SBR $\left(r^{2}=0.26\right)$.

During the blinded visual rating of DAT-SPECT images, at least 1 rater reported a probable abnormality in $4 / 23$ higher risk participants $(17.4 \%)$, compared with $0 / 23$ in the lower risk participants $(P=.109)$, with no difference in "possible" abnormalities (see Supplementary Tables 1 and 2).

A total of 44 participants had at least 1 bone window through which SN-max could be ascertained and measured. SN-max was associated with the MDSUPDRS motor score $\left(r^{2}=0.11, P=.027\right)$ but none of the intermediate markers (UPSIT, RBDSQ, and KS). 
SN-max was also strongly associated with risk estimates $\left(r^{2}=0.27, P<.001\right)$. Higher risk participants had greater mean SN-max than the lower risk participants $\left(0.22 \mathrm{~cm}^{2}\right.$ vs $\left.0.14 \mathrm{~cm}^{2} ; P<.001\right)$, and a greater proportion of higher than lower risk participants had SN hyperechogenicity on blinded rating $(65 \%$ vs $29 \% ; P=.017)$. Multivariate analysis was not undertaken because only 2 significant associations were observed in univariate analysis. There was no evidence of an association between SBR and SN-max $(P=.52)$.

\section{Discussion}

In this study, we report evidence that DAT-SPECT SBR was associated with a range of features of the prediagnostic phase of PD. SBR correlated not only with subtle motor dysfunction using the MDS-UPDRS and finger-tapping speed but also with nonmotor prediagnostic features of PD (lower UPSIT scores and higher RBDSQ scores), and with PREDICT-PD risk estimates, which combine a number of risk and prediagnostic features. This adds further support to the notion that DAT-SPECT is an important marker of nigro-striatal dopamine denervation in the prodromal phase of the disease.

Generalized motor dysfunction (using the MDSUPDRS) and smell loss (using the UPSIT) were most closely associated with SBRs. These features may be manifestations of PD that occur closer to the point of diagnosis than other features, such as the combined PREDICT-PD risk score and subjective RBD, which were more weakly associated with SBR. It is also likely that other neurotransmitter disturbances such as cholinergic dysfunction contribute to these nonmotor features in the earliest stages of PD. ${ }^{10}$

Importantly, although each of the factors studied (risk scores, UPSIT and RBDSQ scores, and motor scores) individually explained some of the variance in DAT-SPECT SBR, combining these features increased their predictive accuracy. It is also noteworthy that the prediction of DAT-SPECT SBR was not improved further by adding MDS-UPDRS motor score, suggesting that the remote assessments can help identify the risk of nigrostriatal dysfunction without the need for in-person assessment.

Strong associations between smell loss and SBR have been reported in the Parkinson's Associated Risk Syndrome (PARS) study, with male gender and constipation identified as additional exposures that predict DAT deficit. ${ }^{11}$ Recently, the PARS investigators reported that the combination of hyposmia and DAT deficit at baseline ultimately predicted new cases of PD during follow-up. ${ }^{1}$ We found similar associations for UPSIT and SBR, but the addition of the RBDSQ, alternate finger-tapping speed, and PREDICT-PD risk scores improved SBR prediction beyond the use of UPSIT in isolation. Similarly, prospective studies of patients with confirmed idiopathic RBD have shown DAT-SPECT to be a strong predictor of those that go on to be diagnosed with PD. ${ }^{2,3}$

The number of individuals undergoing DAT-SPECT in our study was smaller than some of the other studies undertaken in specific risk groups. ${ }^{1,2}$ However, the PREDICT-PD study recruited from the general population, without restriction to any individual risk or prodromal feature such as smell deficit, RBD, or a genetic risk factor, and our finding of an additional contribution of the PREDICT-PD risk score in prediction of SBR suggests that this may be a useful first step in selecting individuals for further objective testing.

SN hyperechogenicity on TCS was associated with MDS-UPDRS motor scores and risk estimates, but not other prediagnostic features. SN hyperechogenicity is thought to be a static marker of PD risk rather than a marker of disease progression. ${ }^{12}$ Similar to previous studies, no association between SN hyperechogenicity and SBR deficit was observed, suggesting that they identify different pathophysiological processes. ${ }^{13,14}$

\section{Limitations}

The study design preferentially included individuals with the highest risk estimates from the PREDICT-PD study. The results may therefore not directly reflect the findings in an unselected older population. Any confounding effect of age, which is included in the risk score, would have been taken account of in the multivariate analysis. There is a chance, however, that a confounding effect of gender may be inadequately adjusted for given the higher proportion of males in the study. Although the interpretation of DAT-SPECT and TCS images used both qualitative and quantitative methods, and the qualitative analysis was blinded, scoring of the MDS-UPDRS was performed by an investigator not blinded to risk status and therefore the possibility of observer bias could not be excluded.

Overall, this study provides support for DATSPECT and TCS as a risk markers for prediagnostic PD and for the potential usefulness of DAT-SPECT in tracking prediagnostic disease progression. We suggest that the combination of being higher risk using the PREDICT-PD algorithm along with subjective RBD, smell loss, and motor impairment are useful in identifying striatal pathology and that this combination is superior to using individual features.

\footnotetext{
Acknowledgments: We thank the staff at the Lysholm Department of Neuroradiology, University College London Hospital, and the staff at the Institute of Nuclear Medicine, University College London for their support in the imaging investigations and infrastructure; Martin Sojer, Heike Stockner and Werner Poewe in Innsbruck, Austria, for their training in the use of transcranial sonography; and the late Selina Paul for her dedication to this project.
} 


\section{References}

1. Jennings D, Siderowf A, Stern M, et al. Conversion to Parkinson disease in the PARS Hyposmic and Dopamine Transporter-Deficit Prodromal Cohort. JAMA Neurol 2017;74(8):933-940.

2. Iranzo A, Santamaria J, Valldeoriola F, et al. Dopamine transporter imaging deficit predicts early transition to synucleinopathy in idiopathic rapid eye movement sleep behavior disorder. Ann Neurol 2017;82(3):419-428.

3. Li Y, Kang W, Yang Q, et al. Predictive markers for early conversion of iRBD to neurodegenerative synucleinopathy diseases. Neurology 2017;88(16):1493-1500.

4. Berg D, Seppi K, Behnke S, et al. Enlarged substantia nigra hyperechogenicity and risk for Parkinson disease: a 37-month 3-center study of 1847 older persons. Arch Neurol 2011;68:932-937.

5. Berg D, Siefker C, Becker G. Echogenicity of the substantia nigra in Parkinson's disease and its relation to clinical findings. J Neurol 2001;248:684-689.

6. Noyce AJ, R'Bibo L, Peress L, et al. PREDICT-PD: an online approach to prospectively identify risk indicators of Parkinson's disease. Mov Disord 2017;32(2):219-226.

7. Noyce AJ, Bestwick JP, Silveira-Moriyama L, et al. Meta-analysis of early nonmotor features and risk factors for Parkinson disease. Ann Neurol 2012;72(6):893-901.

8. Goetz CG, Tilley BC, Shaftman SR, et al. Movement Disorder Society-sponsored revision of the Unified Parkinson's Disease Rating Scale (MDS-UPDRS): scale presentation and clinimetric testing results. Mov Disord 2008;23(15):2129-2170.
9. Soderlund TA, Dickson JC, Prvulovich E, et al. Value of semiquantitative analysis for clinical reporting of 123I-2-beta-carbomethoxy-3beta-(4-iodophenyl)-N-(3-fluoropropyl)nortropane SPECT studies. J Nucl Med 2013;54(5):714-722.

10. Bohnen NI, Albin RL. The cholinergic system and Parkinson disease. Behav Brain Res 2011;221(2):564-573.

11. Jennings D, Siderowf A, Stern M, et al. Imaging prodromal Parkinson disease: the Parkinson Associated Risk Syndrome Study. Neurology 2014;83(19):1739-1746.

12. Berg D, Merz B, Reiners K, Naumann M, Becker G. Five-year follow-up study of hyperechogenicity of the substantia nigra in Parkinson's disease. Mov Disord 2005;20:383-385.

13. Li D-H, Zhang L-Y, Hu Y-Y, et al. Transcranial sonography of the substantia nigra and its correlation with DAT-SPECT in the diagnosis of Parkinson's disease. Parkinsonism Relat Disord 2015; 21:923-928.

14. Bor-Seng-Shu E, Pedroso JL, Felicio AC, et al. Substantia nigra echogenicity and imaging of striatal dopamine transporters in Parkinson's disease: a cross-sectional study. Parkinsonism Relat Disord 2014;20:477-481.

\section{Supporting Data}

Additional Supporting Information may be found in the online version of this article at the publisher's web-site. 